ANNALES

POLONICI MATHEMATICI

$81.1(2003)$

\title{
Estimates for the Bergman kernel and metric of convex domains in $\mathbb{C}^{n}$
}

\author{
by Nikolai Nikolov (Sofia) and Peter Pflug (Oldenburg)
}

\begin{abstract}
Sharp geometrical lower and upper estimates are obtained for the Bergman kernel on the diagonal of a convex domain $D \subset \mathbb{C}^{n}$ which does not contain complex lines. It is also proved that the ratio of the Bergman and Carathéodory metrics of $D$ does not exceed a constant depending only on $n$.
\end{abstract}

1. Introduction. For a domain $D \subset \mathbb{C}^{n}$ we denote by $L_{\mathrm{h}}^{2}(D)$ the Hilbert space of all holomorphic functions $f$ on $D$ that are square-integrable and by $\|f\|_{D}$ the $L^{2}$-norm of $f$. Let $K_{D}$ be the restriction to the diagonal of the Bergman kernel function of $D$. It is well known (cf. [5]) that

$$
K_{D}(a)=\sup \left\{|f(a)|^{2}: f \in L_{\mathrm{h}}^{2}(D),\|f\|_{D} \leq 1\right\} .
$$

If $K_{D}(z)>0$ for some point $z \in D$, then the Bergman metric $B_{D}(z ; X)$, $X \in \mathbb{C}^{n}$, is well defined and can be given by the equality

$$
B_{D}(z ; X)=\frac{M_{D}(z ; X)}{\sqrt{K_{D}(z)}},
$$

where $M_{D}(z ; X)=\sup \left\{\left|f^{\prime}(z) X\right|: f \in L_{\mathrm{h}}^{2}(D),\|f\|_{D}=1, f(z)=0\right\}$.

Let $D \subset \mathbb{C}^{n}$ be a convex domain. If $D$ contains a complex line, then it is linearly equivalent to the Cartesian product of $\mathbb{C}$ and a convex domain in $\mathbb{C}^{n-1}$, which implies that $K_{D} \equiv 0$. Assume now that $D$ does not contain any complex line. Then it is biholomorphically equivalent to a bounded domain (cf. [5]), and hence $K_{D}$ is a positive function on $D$.

For any $a \in D$ fix a point $a^{1} \in \partial D$ such that $d_{1}(a):=\operatorname{dist}(a, \partial D)=$ $\left\|a^{1}-a\right\|$. Observe that $a^{1}$ is not necessarily uniquely defined. Let $H_{1}$ be the complex hyperplane through $a$ which is orthogonal to the vector $a^{1}-a$. Put $D_{1}:=H_{1} \cap D$ and treat $D_{1}$ as an $(n-1)$-dimensional convex subdomain of $H_{1}$. Now, choose an $a^{2} \in \partial D_{1}$ satisfying $d_{2}(a):=\operatorname{dist}\left(a, \partial_{H_{1}} D_{1}\right)=$ $\left\|a_{2}-a\right\|$. Repeating this procedure, we define a sequence of boundary points

2000 Mathematics Subject Classification: Primary 32A25.

Key words and phrases: Bergman kernel, Bergman metric. 
$a^{1}, a^{2}, \ldots, a^{n}$ with $a^{k} \in \partial_{H_{k-1}} D_{k-1}$ (in general, this sequence is not uniquely determined), a sequence of $(n-k)$-dimensional planes $H_{k}, k=1, \ldots, n-1$, through the point $a$, and a sequence of numbers $d_{1}(a), \ldots, d_{n}(a)$ such that $\left\|a^{k}-a\right\|=\operatorname{dist}\left(a, \partial_{H_{k-1}} D_{k-1}\right)=d_{k}(a)$, where $D_{0}:=D$ and $H_{0}:=\mathbb{C}^{n}$. Define

$$
A(a):=d_{1}(a) \ldots d_{n}(a) .
$$

In [3] (cf. also [6], [7]) a similar geometrically defined number $\widetilde{A}(a)$ was introduced and it was proved that if $D$ is a smooth bounded pseudoconvex domain $\mathbb{C}^{n}$ which is convex near a finite-type boundary point $a_{0}$, then there exist positive constants $c$ and $C$ such that $c \leq K_{D} \widetilde{A}^{2}(a) \leq C$ for any $a \in D$ close to $a_{0}$. Also estimates for the Bergman metric were obtained.

The main purpose of this note is to extend these results to any convex domain which does not contain any complex line. Then the results mentioned above follow by a localization argument [4].

TheOREM 1. Let $D \subset \mathbb{C}^{n}$ be a convex domain which does not contain any complex line. Then

$$
\frac{1}{(4 \pi)^{n}} \leq K_{D}(a) A^{2}(a) \leq \frac{(2 n) !}{(2 \pi)^{n}}, \quad a \in D .
$$

Both of these inequalities are sharp. Moreover, the right inequality is always strict iff $n \geq 2$; whereas for all $n \geq 1$, the left inequality can become an equality.

Before stating the second theorem recall that the Carathéodory metric does not exceed the Kobayashi and Bergman metrics, and, in general, there is no relation between the last two metrics (cf. [5]). On the other hand, the Lempert theorem says that the Carathéodory and Kobayashi metrics coincide on any convex domain $D \subset \mathbb{C}^{n}$. Moreover, if $F_{D}$ denotes any of them, if $D$ does not contain the complex line in the direction of $X$, and if

$$
d(z, X):=\sup \{\lambda>0: z+\alpha X \in D \text { if }|\alpha|<\lambda\},
$$

then (see [1])

$$
1 / 2 \leq F_{D}(z ; X) d(z, X) \leq 1 ;
$$

otherwise, $F_{D}(z ; X)=0$.

Similar estimates to those in Theorem 1 are also true for the Bergman metric.

Theorem 2. Let $D \subset \mathbb{C}^{n}$ be a convex domain which does not contain any complex line. Then for any $X \in \mathbb{C}^{n} \backslash\{0\}$ we have

$$
\frac{1}{2} \leq B_{D}(z ; X) d(z, X) \leq n \sqrt{\frac{2^{n-1}(2(n+1)) !}{3}} .
$$


In particular,

$$
1 \leq \frac{B_{D}(z ; X)}{F_{D}(z ; X)} \leq 2 n \sqrt{\frac{2^{n-1}(2(n+1)) !}{3}} .
$$

Note that if $c_{n}$ and $C_{n}$ are the best constants for which the last inequality in Theorem 2 holds, then $c_{n} \leq \sqrt{2}$ and $C_{n} \geq \sqrt{n+1}$ as the examples of the unit polydisc and the unit ball show. On the other hand, except the whole complex plane, every convex plane domain is biholomorphically equivalent to the unit disc, which shows that $c_{1}=C_{1}=\sqrt{2}$.

Remark. Observe that Theorem 2 covers some of the results in [8].

2. Proofs. Using some ideas from [6], we shall give purely geometrical proofs, based on the transformation rules for $K_{D}$ and $M_{D}$, and the decreasing property of these invariants under inclusion of domains.

Proof of Theorem 1. Let $a \in D, A:=A(a)$, and $d_{k}:=d_{k}(a), 1 \leq k \leq n$. After a translation and a unitary transformation we may assume that $a=0$, $a^{1}=\left(d_{1}, 0, \ldots, 0\right), H_{1}=\left\{z_{1}=0\right\}, a^{2}=\left(0, d_{2}, 0, \ldots, 0\right), H_{2}=\left\{z_{1}=z_{2}=0\right\}$, $\ldots, a^{n}=\left(0, \ldots, 0, d_{n}\right)$. Since $D$ is convex, it contains the domain

$$
G:=\left\{z \in \mathbb{C}^{n}: \sum_{j=1}^{n}\left|z_{j}\right| / d_{j}<1\right\} .
$$

Let $\mathbb{B}\left(0, d_{1}\right)$ denote the ball in $\mathbb{C}^{n}$ with center at 0 and radius $d_{1}$. Then $G \cup \mathbb{B}\left(0, d_{1}\right) \subset D$ and hence

$$
K_{D}(0) \leq K_{G \cup \mathbb{B}\left(0, d_{1}\right)}(0) \leq K_{G}(0)=K_{E}(0) / A^{2},
$$

where

$$
E:=\left\{z \in \mathbb{C}^{n}: \sum_{j=1}^{n}\left|z_{j}\right|<1\right\} .
$$

Observe that $K_{E}(0)=(2 n) ! /(2 \pi)^{n}$.

Note that if $n \geq 2$, then $G$ does not contain $\mathbb{B}\left(0, d_{1}\right)$; thus the second inequality above is strict (recall that for a complete Reinhardt domain $\widetilde{G}$ we have $\left.K_{\widetilde{G}}(0)=\operatorname{vol}(\widetilde{G})^{-1}\right)$.

To prove the left inequality, let

$$
S_{k}:=\left\{z \in \mathbb{C}^{n}: \operatorname{Re} \sum_{j=1}^{n} \alpha_{k j} z_{j}=\operatorname{Re}\left(\alpha_{k k} d_{k}\right)\right\}
$$

be a supporting hyperplane for $\partial D$ at $a^{k}$. By our construction it follows that $\alpha_{k j}=0$ for $j>k$, and $\alpha_{k k}$ is a non-zero real number; we may assume that $\alpha_{k k}=1$. Set 


$$
\varphi_{k}(z):=\sum_{j=1}^{k} \alpha_{k j} z_{j}-d_{k}, \quad P_{k}:=\left\{z \in \mathbb{C}^{n}: \operatorname{Re} \varphi_{k}(z)<0\right\} .
$$

Since $D$ is convex, it follows that $D \subset P:=\bigcap_{j=1}^{n} P_{j}$. Note that $\Phi(z):=$ $\left(\varphi_{1}(z), \ldots, \varphi_{n}(z)\right)$ is an affine mapping with jacobian 1 that transforms $P$ into the Cartesian product $\Pi^{n}$ of $n$ copies of the left half-plane $\Pi$ and the origin into the point $\left(-d_{1}, \ldots,-d_{n}\right)$. Hence

$$
K_{D}(0) \geq K_{P}(0)=\prod_{j=1}^{n} K_{\Pi}\left(-d_{j}\right)=\frac{1}{(4 \pi)^{n} A^{2}} .
$$

To see that the left inequality is sharp, it suffices to take $D=\Pi^{n}$.

If $n=1$, taking $D$ to be the unit disc we see that the right inequality is sharp. Let $n \geq 2, m$ a positive integer, and $b_{k}=k^{m}$ for $1 \leq k \leq n$. Let $B_{k}:=\mathbb{B}\left(0, b_{k}\right) \cap H_{k-1}$. Define $\widehat{D}$ to be the convex envelope of the union of $\bigcup_{k=1}^{n} B_{k}$ and $\left\{z \in \mathbb{C}^{n}: \sum_{k=1}^{n}\left|z_{k}\right| / b_{k}<1\right\}$. It is not difficult to see that $\widehat{D}$ is a convex domain and $b_{k}=\operatorname{dist}\left(0, \partial\left(\widehat{D} \cap H_{k-1}\right)\right)$.

If $\Psi(z):=\left(z_{1} / b_{1}, \ldots, z_{n} / b_{n}\right)$, then $\Psi(\widehat{D})$ is the convex envelope of the union of $S:=\bigcup_{k=1}^{n} \Psi\left(B_{k}\right)$ and $E$. For any $j>k$ we have $b_{j} / b_{k} \rightarrow \infty$ as $m \rightarrow \infty$. Hence, for any $\lambda>1$ we may find an $m$ such that $S \subset \lambda E$. Since $\lambda E$ is convex, it contains $\Psi(\widehat{D})$. Hence

$$
K_{\widehat{D}}(0)\left(b_{1} \ldots b_{n}\right)^{2}=K_{\Psi(\widehat{D})}(0) \geq K_{\lambda E}(0)=K_{E}(0) / \lambda^{2 n},
$$

which completes the proof of the theorem.

Proof of Theorem 2. We only have to prove the right inequality. We shall use the notations from Theorem 1 and the geometric setting in the proof of Theorem 1, in particular $a=0$. Let $X=\left(X_{1}, \ldots, X_{n}\right) \in \mathbb{C}^{n} \backslash\{0\}$ and fix $k \in J:=\left\{j: X_{j} \neq 0\right\}$. Then

$\Psi_{k}(z):=\left(z_{1}-\frac{X_{1}}{X_{k}} z_{k}, \ldots, z_{k-1}-\frac{X_{k-1}}{X_{k}} z_{k}, z_{k}, z_{k+1}-\frac{X_{k+1}}{X_{k}} z_{k}, \ldots, z_{n}-\frac{X_{n}}{X_{k}} z_{k}\right)$

is a linear mapping with jacobian equal to 1 and $Y^{k}:=\Psi_{k}(X)=\left(0, \ldots, 0, X_{k}\right.$, $0, \ldots, 0)$. Let $\Delta_{j}^{\prime}:=\{0\} \times \ldots \times\{0\} \times \Delta_{j} \times\{0\} \times \ldots \times\{0\}$ be the disc in the $j$ th coordinate plane with center at the origin and radius $d_{j}$ if $j \neq k$, and $d_{k}^{\prime}:=\left|X_{k}\right| d(0, X)$ if $j=k$. It is easy to see that $\Delta_{j}^{\prime} \subset G_{k}:=\Psi_{k}(D)$. Since $G_{k}$ is convex, it contains the domain

$$
G_{k}^{\prime}=\left\{z \in \mathbb{C}^{n}: \frac{\left|z_{k}\right|}{d_{k}^{\prime}}+\sum_{j=1, j \neq k}^{n} \frac{\left|z_{j}\right|}{d_{j}}<1\right\}
$$

Hence

$$
M_{D}(0 ; X)=M_{G_{k}}\left(0 ; Y^{k}\right) \leq M_{G_{k}^{\prime}}\left(0 ; Y^{k}\right)=C \frac{d_{k}}{A\left|X_{k}\right| d^{2}(0, X)},
$$


where $C:=M_{E}\left(0 ; e_{1}\right)$ and $e_{1}=(1,0, \ldots, 0)$. Applying the left inequality of Theorem 1 and using the equality $d_{k}=\left|X_{k}\right| d\left(0, Y^{k}\right)$, we obtain

$$
B_{D}(0 ; X)=\frac{M_{D}(0 ; X)}{\sqrt{K_{D}(0)}} \leq C(2 \sqrt{\pi})^{n} \frac{d\left(0, Y^{k}\right)}{d^{2}(0, X)} .
$$

On the other hand, by the formula for $K_{E}$ in [9] (cf. also [2] and [5]), it follows that $\left({ }^{1}\right)$

$$
K_{E}\left(\left(z_{1}, 0^{\prime}\right)\right)=\frac{(2 n-1) !}{(2 \pi)^{n}} \frac{1}{2\left|z_{1}\right|}\left(\frac{1}{\left(1-\left|z_{1}\right|\right)^{2 n}}-\frac{1}{\left(1+\left|z_{1}\right|\right)^{2 n}}\right) .
$$

Since

$$
B_{E}\left(0 ; e_{1}\right)=\sqrt{\left.\frac{\partial^{2}}{\partial z_{1} \partial \bar{z}_{1}} \log K_{E}\left(\left(z_{1}, 0^{\prime}\right)\right)\right|_{z_{1}=0}},
$$

we find that $C=\sqrt{(2(n+1)) ! /\left(6(2 \pi)^{n}\right)}$.

Finally, observe that

$$
\frac{1}{d(0, X)} \leq \sum_{s \in J} \frac{1}{d\left(0, Y^{s}\right)}
$$

(since $D$ is convex). It remains to compare the last two inequalities. Hence the proof of Theorem 2 is finished.

Acknowledgments. This paper was written during the stay of the authors at the Mathematical Institute of the Jagiellonian University in Kraków, Poland. Both of us wish to thank all the colleagues there for their great hospitality.

\section{References}

[1] E. Bedford and S. I. Pinchuk, Convex domains with noncompact groups of automorphisms, Russian Acad. Sci. Sb. Math. 82 (1995), 1-20.

[2] H. P. Boas, S. Fu and E. J. Straube, The Bergman kernel function: explicit formulas and zeroes, Proc. Amer. Math. Soc. 127 (1999), 805-811.

[3] J.-H. Chen, Estimates of the invariant metrics on convex domains in $\mathbb{C}^{n}$, thesis, Purdue Univ., 1989.

[4] K. Diederich, J. E. Fornæss and G. Herbort, Boundary behavior of the Bergman metric, in: Proc. Sympos. Pure Math. 41, Amer. Math. Soc., 1984, 59-67.

[5] M. Jarnicki and P. Pflug, Invariant Distances and Metrics in Complex Analysis, de Gruyter, Berlin, 1993.

[6] J. D. McNeal, Bergman kernels of convex domains, Adv. Math. 109 (1994), 108-139.

[7] - Invariant metric estimates for $\bar{\partial}$ on some pseudoconvex domains, Ark. Mat. 39 (2001), 121-136.

$\left({ }^{1}\right)$ Observe that this function, although formally not defined at $z_{1}=0$, extends smoothly through $z_{1}=0$. 
[8] N. Nikolov and P. Pflug, Behavior of the Bergman kernel and metric near convex boundary points, Proc. Amer. Math. Soc., to appear.

[9] B. S. Zinov'ev, On the reproducing kernels for multicircular domains of holomorphy, Sibirsk. Mat. Zh. 15 (1974), 35-48 (in Russian).

Institute of Mathematics and Informatics Bulgarian Academy of Sciences 1113 Sofia, Bulgaria

E-mail:nik@math.bas.bg
Fachbereich Mathematik Carl von Ossietzky Universität Oldenburg Postfach 2503 D-26111 Oldenburg, Germany E-mail: pflug@mathematik.uni-oldenburg.de

Reçu par la Rédaction le 11.3.2002

Révisé le 3.6.2002 